\title{
Efeito do controle da placa supragengival sobre a microflora subgengival e tecidos periodontais
}

\section{Effect of supragingival plaque control on subgingival microflora and periodontal tissues}

\author{
Allyson Nogueira MOREIRA* \\ Liliana Fernandez CANIGGIA** \\ Raquel Conceição FERREIRA*** \\ Chiappe VERÓNICA**** \\ Carlos ALONSO***** \\ Susana PIOVANO******
}

\begin{abstract}
MOREIRA, A. N.; CANIGGIA, L. F.; FERREIRA, R. C.; VERÓNICA, C.; ALONSO, C.; PIOVANO, S. Efeito do controle da placa supragengival sobre a microflora subgengival e tecidos periodontais. Pesqui Odontol Bras, v. 15, n. 2, p. 119-126, abr./jun. 2001.
\end{abstract}

\begin{abstract}
O objetivo deste trabalho foi estudar, clínica e microbiologicamente, 44 sítios em 11 pacientes com periodontite crônica generalizada. IP, IG, SS, PS e NI foram registrados. Amostras de placa subgengival foram colhidas nos mesmos sítios para cultivo de bactérias anaeróbias e determinação dos morfotipos microbianos por MCE. Os registros clínicos e estudos microbiológicos foram tomados no "baseline" e 4 semanas após a incorporação em um programa de controle de placa e cálculo supragengival. A análise microbiológica categorizou o grau de desenvolvimento em: 0 - não detectado, 1 - escasso, 2 - moderado e 3 - abundante. Os registros clínicos no "baseline" e dia 28 foram: IP - 1,73 $\pm 0,10$ e 0,30 $\pm 0,08, \mathrm{IG}-1,73 \pm 0,08$ e $1,41 \pm 0,08, \mathrm{SS}-0,91 \pm 0,04$ e 0,59 $\pm 0,07, \mathrm{PS}-6,43 \pm 0,20$ e $5,77 \pm 0,25, \mathrm{NI}-6,86 \pm 0,32$ e $6,52 \pm 0,34$, respectivamente. A redução do IP, IG, SS e PS foi significativa. Não foram registradas diferenças significativas no NI. As proporções relativas dos morfotipos bacterianos observados por MCE no "baseline" e dia 28 foram: células cocóideas - 21,16 $\pm 3,77$ e 36,00 \pm 4,66, bacilos móveis - 44,86 $\pm 2,65$ e 39,50 $\pm 2,64$, treponemas totais - 24,66 \pm 3,08 e 19,25 2,75. No "baseline" e no dia 28 foi observado: Pi/n - 1,36 $\pm 0,18$ e 0,43 $\pm 0,11, \mathrm{Pg}-0,48 \pm 0,16$ e 0,32 \pm 0,13, Aa - 0,23 $\pm 0,09$ e 0,23 $\pm 0,10$, Fusobacterium nucleatum - 0,32 $\pm 0,14$ e 0,41 $\pm 0,13$ e peptostreptococos - 0,82 \pm 0,19 e $0,54 \pm 0,16$, respectivamente. Houve um aumento significativo das células cocóideas, diminuição de treponemas e de $\mathrm{Pi} / \mathrm{n}$.
\end{abstract}

UNITERMOS: Periodontia; Microbiologia.

\section{INTRODUÇÃO}

Numerosos investigadores demonstraram a importância dos microorganismos na iniciação e progressão da enfermidade periodontal ${ }^{12,22,24,27}$.

Testes microbiológicos foram aplicados no reconhecimento dos microorganismos associados com saúde e enfermidade, bem como do efeito do tratamento periodontal sobre eles e $^{3-6,8-11,13,14,16,17,18,22-25}$.

O controle da placa supragengival tem sido efetivo na resolução da gengivite ${ }^{12}$.
Desse modo, numerosos estudos clínicos registraram que, em pacientes com enfermidade periodontal, o controle da placa supragengival e subgengival resolveu geralmente a inflamação e limitou a progressão da enfermidade ${ }^{1,2,17,21}$.

Existe controvérsia quanto a influência do controle da placa supragengival sobre a composição da microflora subgengival em sítios enfermos com um ecossistema estabelecido ${ }^{1-4,6,14,25}$.

Em pacientes com profundidade a sondagem

\footnotetext{
*Professor Adjunto; ***Professora Substituta - Faculdade de Odontologia da UFMG.

**Bioquímica, Especialista em Microbiologia.

${ }^{* * * *}$ Chefe de Trabalhos Práticos de Periodontia; $* * * *$ Professor Titular de Periodontia - Faculdade de Odontologia da Universidade de Buenos Aires.

$* * * * * *$ Diretora de Investigação da Faculdade de Odontologia da Universidade de Maimónides e Professora Adjunta da Faculdade de Odontologia da Universidade de Buenos Aires.
} 
MOREIRA, A. N.; CANIGGIA, L. F.; FERREIRA, R. C.; VERÓNICA, C.; ALONSO, C.; PIOVANO, S. Efeito do controle da placa supragengival sobre a microflora subgengival e tecidos periodontais. Pesqui Odontol Bras, v. 15, n. 2, p. 119-126, abr./jun. 2001.

(PS) de 4-5 mm, o controle de placa supragengival alterou a microflora subgengival; enquanto, com PS maiores, os efeitos não foram observados ou foram menores ${ }^{3,4,6,14}$.

O objetivo deste trabalho foi analisar o efeito do controle da placa supragengival sobre a composição da microflora subgengival e sobre os tecidos periodontais em pacientes com diagnóstico de periodontite crônica generalizada.

\section{MATERIAL E MÉTODOS}

Foram selecionados 11 pacientes entre 35 e 60 anos ( $\bar{x} 50,3 \pm 2,59$ ), de ambos sexos, com pelo menos 20 dentes presentes com diagnóstico de periodontite crônica generalizada e PS $\geq 5 \mathrm{~mm}^{14,19}$.

\section{Critérios de exclusão}

Foram excluídos os pacientes que receberam tratamento periodontal nos últimos 6 meses e antibióticos ou imunosupressores nos 3 meses prévios ao estudo. Outros critérios de exclusão: diabetes, gravidez-lactação, quadros periodontais agudos (abscessos periodontais e gengivite úlcero-necrosante aguda - GUNA).

\section{Indicadores clínicos}

Foram incluídos os índices de placa ${ }^{12}$, gengi$\mathrm{val}^{12}$, sangramento a sondagem, profundidade a sondagem $^{20}$ e nivel clínico de inserção. Foram observadas as superficies distal, vestibular, mesial e palatina ou lingual de todos os dentes presentes, utilizando sonda periodontal*.

\section{Estudo microbiológico}

Em cada paciente, foram selecionados 4 sítios com PS $\geq 5 \mathrm{~mm}$ para estudo microbiológico (um por quadrante). Nos sítios selecionados, a placa supragengival foi removida com cureta periodontal Gracey $n^{\circ} 5,6^{*}$ e descartada.

\section{Coleta da placa subgengival}

\section{Para cultivo}

Foram colocados dois cones de papel ${ }^{* *} \mathrm{n}^{\circ} 30 \mathrm{e}$ 35 nos sítios selecionados e deixados em contato durante 15 segundos. Após este período, foram retirados e colocados em um tubo contendo meio de transporte VMGA III ${ }^{15}$.

*Hu Friedy.

**Johnson \& Johnson.
Para observação em Microscópio de Campo Escuro (MCE)

Dando continuidade à coleta anterior, foram tomadas amostras de placa subgengival com cureta periodontal Gracey $\mathrm{n}^{\circ}$ 5, 6 e colocadas em 0,3 ml de solução fisiológica mais gelatina a $1 \%{ }^{8}$. Todas as amostras, identificadas com o nome do paciente e o sítio da coleta, foram remetidas ao laboratório dentro das duas primeiras horas.

\section{Processamento das amostras}

Foram realizados exame direto e cultivo com identificação.

\section{Exame direto}

$\mathrm{Da}$ amostra suspensa em solução fisiológica mais gelatina a $1 \%, 0,1 \mathrm{ml}$ foi retirado e colocado em uma lâmina de vidro e coberto por uma lamínula. Os morfotipos microbianos foram examinados por MCE segundo LISTGARTEN; HELLDEN ${ }^{10}$ (1978), LISTGARTEN; SCHIFTER ${ }^{11}$ (1982) e LISTGARTEN $^{9}$ (1984) e interpretados segundo MOUSQUES et al. ${ }^{17}$ (1980) e MOUSQUES et al. ${ }^{18}$ (1980).

\section{Cultivo com identificação}

As amostras para cultivo foram semeadas em meios seletivos e não seletivos para a recuperação de bactérias anaeróbias e anaeróbias facultativas segundo SUMMANEN et al. ${ }^{26}$ (1993).

Da amostra em meio de transporte VMGA III, foi semeado $0,1 \mathrm{ml}$ por esgotamento em estrias em cada um dos seguintes meios:

1. ágar chocolate base Columbia, para isolamento de microorganismos anaeróbios facultativos;

2. ágar tripticasa soja, bacitracina, vancomicina (TSBV), para isolamento de Actinobacillus actinomycetemcomitans ${ }^{23}$;

3. ágar brucella suplementado com extratos de levedura $(10 \mu \mathrm{g} / \mathrm{ml})$, hemina $(5 \mu \mathrm{g} / \mathrm{ml})$, vitamina $\mathrm{K}(1 \mu \mathrm{g} / \mathrm{ml})$ e sangue $(5 \%)(\mathrm{ABS})$;

4. ágar brucella sangue com o agregado de amicacina $(5 \mu \mathrm{g} / \mathrm{ml})$ e colistina $(10 \mu \mathrm{g} / \mathrm{ml})$ (ABSAC).

As placas de ágar chocolate base Columbia e TSBV foram incubadas a $37^{\circ} \mathrm{C}$ em atmosfera enriquecida de $\mathrm{CO}_{2}(10 \%)$ durante 7 dias com leituras a cada 48 horas.

As placas ABS e ABSAC foram incubadas a $37^{\circ} \mathrm{C}$ em câmara anaeróbia durante 14 dias em atmosfera de $5 \%$ de $\mathrm{H}_{2}, 5 \%$ de $\mathrm{CO}_{2}, 90 \%$ de $\mathrm{N}_{2}$ e foram observadas nos dias 5,10 e $14^{25}$. 
MOREIRA, A. N.; CANIGGIA, L. F.; FERREIRA, R. C.; VERÓNICA, C.; ALONSO, C.; PIOVANO, S. Efeito do controle da placa supragengival sobre a microflora subgengival e tecidos periodontais. Pesqui Odontol Bras, v. 15, n. 2, p. 119-126, abr./jun. 2001.

O grau de desenvolvimento microbiano foi categorizado usando o seguinte "score":

0 - desenvolvimento não detectado;

1 - desenvolvimento somente na primeira estria (escasso);

2 - desenvolvimento na primeira e segunda estria (moderado);

3 - desenvolvimento até a terceira e/ou quarta estria (abundante).

Os microorganismos aeróbios selecionados foram identificados por provas bioquímicas convencionais e as bactérias anaeróbias por provas bioquímicas e detecção de enzimas pré-formadas utilizando-se substratos fluorgênicos.

\section{Desenho experimental}

Os exames clínico-microbiológicos foram realizados nos dias 0 e 28 .

No dia zero, realizados os exames clínico-microbiológicos, os pacientes foram introduzidos em um programa de controle de placa e cálculo supragengival que incluiu:

1. técnicas de higiene bucal com escova dental e elementos interdentais;

2. controle profissional de placa e cálculo supragengival mediante raspagem supragengival mecânica com cureta periodontal Gracey $n^{\circ} 5,6$ ou a utilização de aparelho ultra-sônico* nos dias 7,14 e 21 . No dia 28, os mesmos exames clínico-microbiológicos foram realizados novamente.

\section{Análise estatística}

A média aritmética e o desvio-padrão dos indicadores clínicos obtidos nos 44 sítios analisados microbiologicamente foram realizados. Também foram realizados para os morfotipos microbianos e microorganismos identificados.

O teste $t$ de Student foi utilizado para analisar as variações clínicas e microbiológicas entre a condição basal e o dia 28.

Uma probabilidade de $\mathrm{p}<0,05$ foi aceita para considerar diferenças significativas ${ }^{7}$.

Foi realizada distribuição de freqüência relativa dos indicadores clínicos e microbiológicos na condição basal e dia 28.

\section{Cultivo}

O grau de desenvolvimento dos microorganismos foi categorizado de acordo com os seguintes "scores": 0 - não detectado, 1 - desenvolvimento escasso, 2 - desenvolvimento moderado, 3 - desenvolvimento abundante.

\section{Indicadores clínicos}

Os "scores" considerados para os índices empregados foram: IP - 0, 1, 2, 3; IG - 0, 1, 2, 3; SS - 0, 1; PS - $5 \mathrm{~mm}$, 6-7 mm, > $7 \mathrm{~mm}$.

\section{Microscopia de campo escuro}

Quanto à porcentagem de células cocóideas, utilizou-se a seguinte classificação: 1) $\leq 25 \%$, 2) > $25 \% \leq 50 \%, 3)>50 \%$.

Quanto à porcentagem de treponemas pequenos, medianos, grandes e totais, empregou-se a classificação: 1) < 2\%, 2) $\geq 2 \%<15 \%$, 3) $\geq 15 \%$.

Com a distribuição de freqüência relativa, foi realizado um gráfico de barras e intervalos de confiança com $95 \%$ de segurança ${ }^{7}$.

\section{RESULTADOS}

A Tabela 1 mostra os indicadores clínicos dos sítios analisados na condição basal e no dia 28. No final do período experimental, foi registrada uma diminuição significativa no IP, IG, PS e SS. Não foram observadas diferenças no NI.

As proporções relativas dos morfotipos microbianos da placa subgengival são observados na Tabela 2. No dia 28, comparado à condição basal, foi

TABELA 1 - Parâmetros clínicos dos sítios com programa de controle de placa supragengival $(\mathrm{n}=44)$.

\begin{tabular}{l|c|c|c|c|c}
\hline \hline & Índice de placa (IP) & Índice gengival (IG) & $\begin{array}{c}\text { Sangramento a } \\
\text { sondagem (SS) }\end{array}$ & $\begin{array}{c}\text { Profundidade a } \\
\text { sondagem (PS) }\end{array}$ & $\begin{array}{c}\text { Nível de } \\
\text { inserção (NI) }\end{array}$ \\
\hline Dia 0* & $1,73 \pm 0,10$ & $1,73 \pm 0,08$ & $0,91 \pm 0,04$ & $6,43 \pm 0,20$ & $6,86 \pm 0,32$ \\
\hline & $\mathrm{p}<0,01$ & $\mathrm{p}<0,01$ & $\mathrm{p}<0,001$ & $\mathrm{p}<0,05$ & N.S. \\
\hline Dia 28* & $0,30 \pm 0,08$ & $1,41 \pm 0,08$ & $0,59 \pm 0,07$ & $5,77 \pm 0,25$ & $6,52 \pm 0,34$ \\
\hline \hline
\end{tabular}

N.S. = não significante. *Média aritmética e erro-padrão: diferenças analisadas por teste $t$ de Student.

${ }^{*}$ Le Clean Machine ${ }^{\circledR}$ - Parkell Eletronics Division - NY, EUA. 
MOREIRA, A. N.; CANIGGIA, L. F.; FERREIRA, R. C.; VERÓNICA, C.; ALONSO, C.; PIOVANO, S. Efeito do controle da placa supragengival sobre a microflora subgengival e tecidos periodontais. Pesqui Odontol Bras, v. 15, n. 2, p. 119-126, abr./jun. 2001.

TABELA 2 - Proporções relativas de morfotipos microbianos em sítios com programa de controle de placa supragengi$\operatorname{val}(\mathrm{n}=44)$.

\begin{tabular}{l|c|c|c|c|c|c}
\hline \hline & $\begin{array}{c}\text { Células } \\
\text { cocóideas }\end{array}$ & $\begin{array}{c}\text { Bacilos } \\
\text { móveis }\end{array}$ & $\begin{array}{c}\text { Treponemas } \\
\text { pequenos }\end{array}$ & $\begin{array}{c}\text { Treponemas } \\
\text { medianos }\end{array}$ & $\begin{array}{c}\text { Treponemas } \\
\text { grandes }\end{array}$ & $\begin{array}{c}\text { Treponemas } \\
\text { totais }\end{array}$ \\
\hline Dia $0^{*}$ & $21,16 \pm 3,77$ & $44,86 \pm 2,65$ & $5,39 \pm 1,22$ & $18,45 \pm 2,74$ & $0,27 \pm 0,15$ & $24,66 \pm 3,08$ \\
\hline & $\mathrm{p}<0,05$ & N.S. & $\mathrm{p}<0,05$ & N.S. & N.S. & N.S. \\
\hline Dia $28^{*}$ & $36,00 \pm 4,63$ & $39,50 \pm 2,64$ & $2,30 \pm 0,54$ & $16,77 \pm 2,86$ & $0,09 \pm 0,04$ & $19,25 \pm 2,75$ \\
\hline \hline
\end{tabular}

N.S. = não significante. *Média aritmética e erro-padrão: diferenças analisadas por teste $t$ de Student.

TABELA 3 - Grau de desenvolvimento de microorganismos prevalentes em sítios com programa de controle de placa supragengival $(\mathrm{n}=44)$.

\begin{tabular}{l|c|c|c|c|c}
\hline \hline & $\begin{array}{c}\text { P. intermedial } \\
\text { nigrescens }\end{array}$ & P. gingivalis & $\begin{array}{c}\text { A. actinomyce- } \\
\text { temcomitans }\end{array}$ & Fusobacterium & Peptostreptococos \\
\hline Dia $0^{*}$ & $1,36 \pm 0,18$ & $0,48 \pm 0,16$ & $0,23 \pm 0,09$ & $0,32 \pm 0,14$ & $0,82 \pm 0,19$ \\
\hline & $\mathrm{p}<0,001$ & N.S. & N.S. & N.S. & N.S. \\
\hline Dia $28^{*}$ & $0,43 \pm 0,11$ & $0,32 \pm 0,13$ & $0,23 \pm 0,10$ & $0,41 \pm 0,13$ & $0,54 \pm 0,16$ \\
\hline \hline
\end{tabular}

N.S. = não significante. *Média aritmética e desvio-padrão: diferenças analisadas por teste $t$ de Student.

registrado aumento significativo de células cocóideas e diminuição significativa de treponemas pequenos, enquanto não se observaram diferenças nos bacilos móveis, treponemas medianos, grandes e totais.

Nos microorganismos identificados por cultivo, no dia 28, comparado com a condição basal, foi registrado: diminuição significativa no grau de desenvolvimento de Prevotella intermedia/nigrescens. Para os demais microorganismos estudados, não foram observadas diferenças significativas (Tabela 3).

O índice de placa, na condição basal, evidenciou prevalência do grau 2 , enquanto, no final do período experimental, foi registrada uma diminuição do grau 2 e uma maior prevalência do índice de placa grau 0 (Gráfico 1).

O índice gengival, no final do período experimental, registrou uma tendência a diminuição do grau 2 e um aumento das proporções de sítios com grau 1 (Gráfico 2). O índice SS registrou uma tendência a diminuir, pois houve uma redução do sangramento (Gráfico 3).

A distribuição da freqüência relativa dos indicadores clínicos evidenciou, no final do período experimental, uma diminuição nos sítios de 6-7 mm e um aumento nas proporções de sítios com $5 \mathrm{~mm}$ (Gráfico 4).

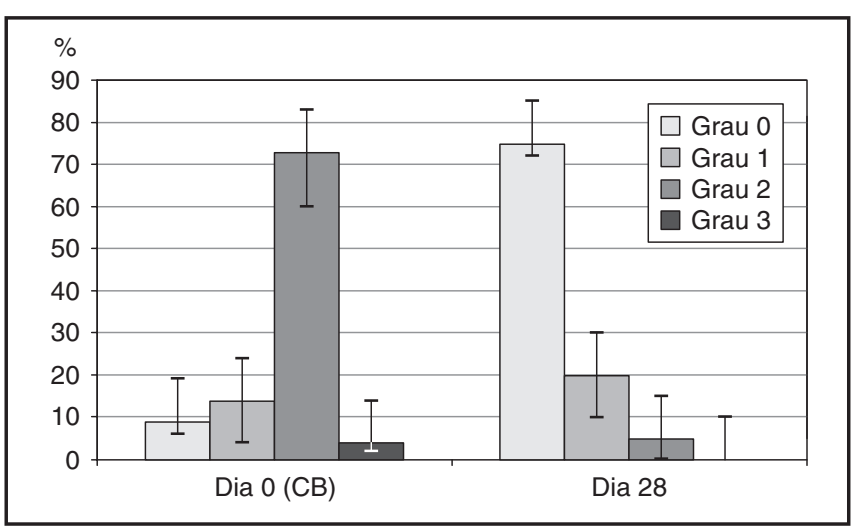

GRÁFICO 1 - Distribuição da freqüência do índice de placa nos períodos experimentais. $\mathrm{CB}=$ condição basal.

Foi evidenciada na análise da distribuição de freqüência relativa dos morfotipos microbianos que as células cocóideas, no final do período experimental, mostraram tendência a diminuição dos categorizados dentro de $\leq 25 \%$ e uma tendência a aumento da distribuição > 50\% (Gráfico 5).

Não foram registradas mudanças nos bacilos móveis e treponemas grandes.

Nos treponemas medianos, foi registrada uma leve tendência à diminuição na proporção dos sítios que registraram $\geq 2 \%<15 \%$ na condição basal e um aumento nos sítios com $<2 \%$. Os treponemas pequenos, no final do período experimental, regis- 
MOREIRA, A. N.; CANIGGIA, L. F.; FERREIRA, R. C.; VERÓNICA, C.; ALONSO, C.; PIOVANO, S. Efeito do controle da placa supragengival sobre a microflora subgengival e tecidos periodontais. Pesqui Odontol Bras, v. 15, n. 2, p. 119-126, abr./jun. 2001.

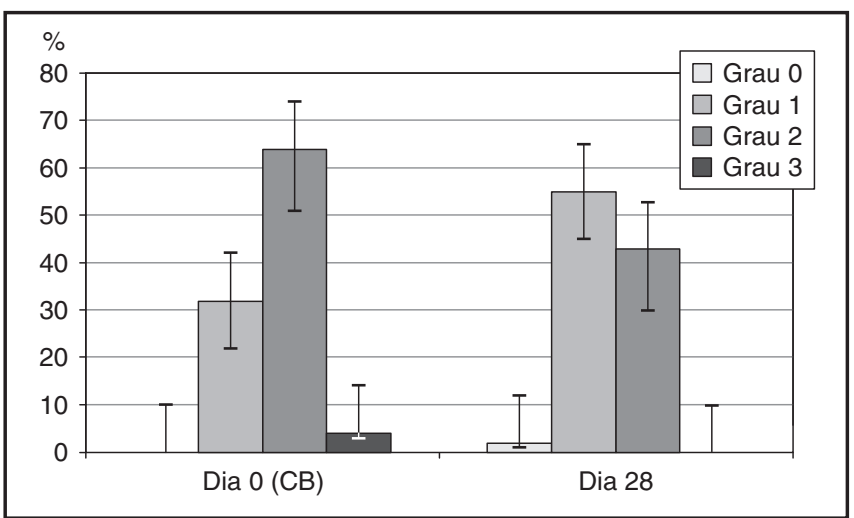

GRÁFICO 2 - Distribuição da freqüência do índice gengival nos períodos experimentais. $\mathrm{CB}=$ condição basal.

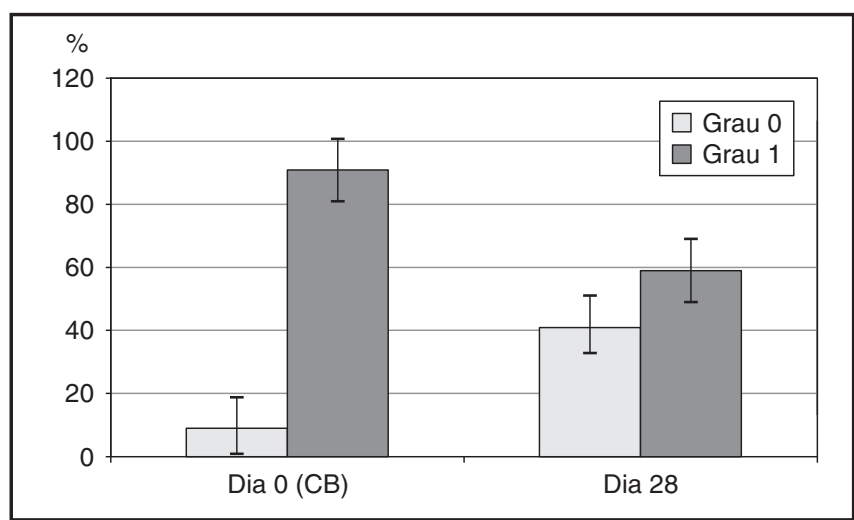

GRÁFICO 3 - Distribuição da freqüência de sangramento a sondagem nos periodos experimentais. $\mathrm{CB}=$ condição basal.

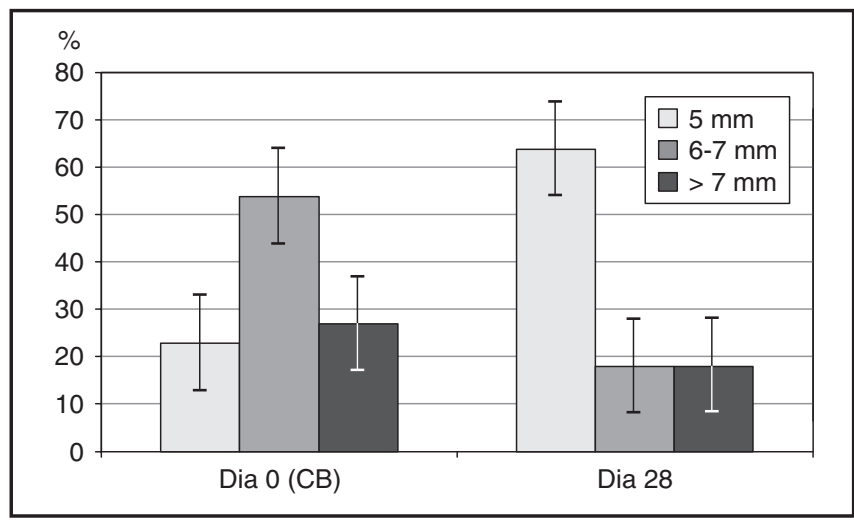

GRÁFICO 4 - Distribuição de freqüência da profundidade a sondagem nos períodos experimentais. $\mathrm{CB}=$ condição basal.

traram ausência de sítios com proporções $\geq 15 \%$ e uma tendência a aumento nos sítios com proporções $<2 \%$ (Gráfico 6).

Com referência ao cultivo, $\mathrm{Pi} / \mathrm{n}$ registrou uma

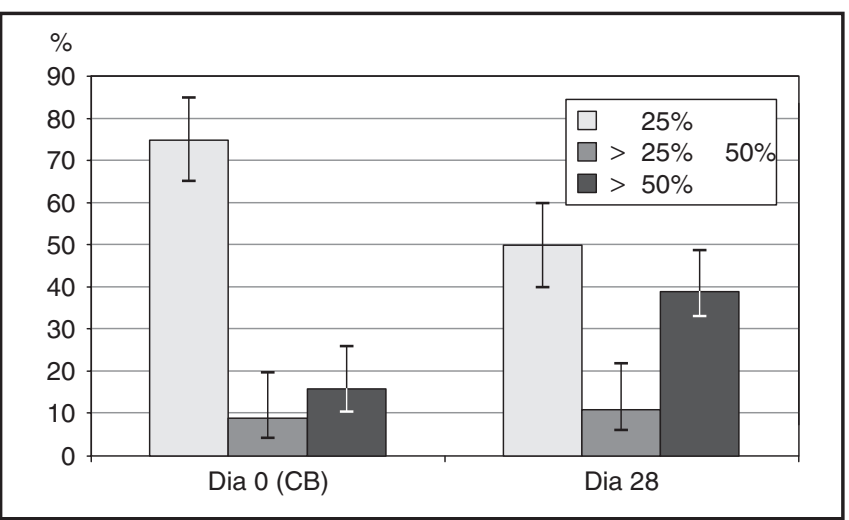

GRÁFICO 5 - Distribuição da freqüência de morfotipos microbianos da placa subgengival (células cocóideas). $\mathrm{CB}=$ condição basal.

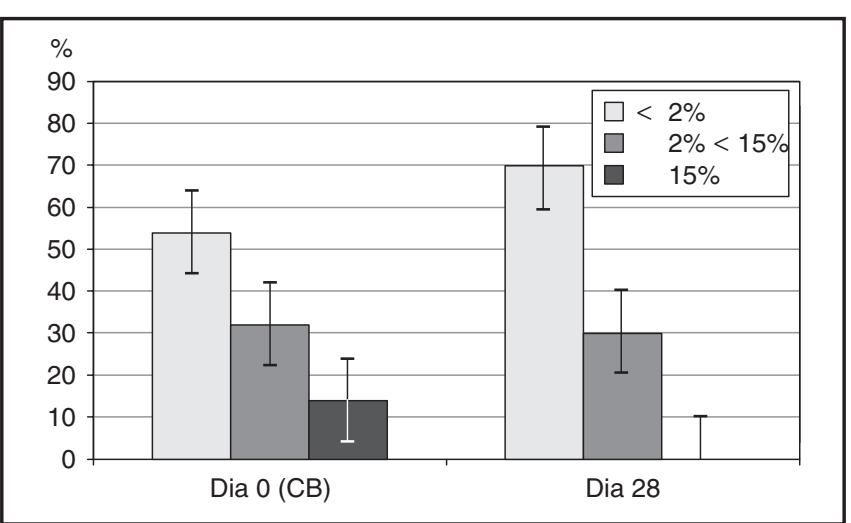

GRÁFICO 6 - Distribuição da freqüência de morfotipos microbianos da placa subgengival (treponemas pequenos). $\mathrm{CB}=$ condição basal.

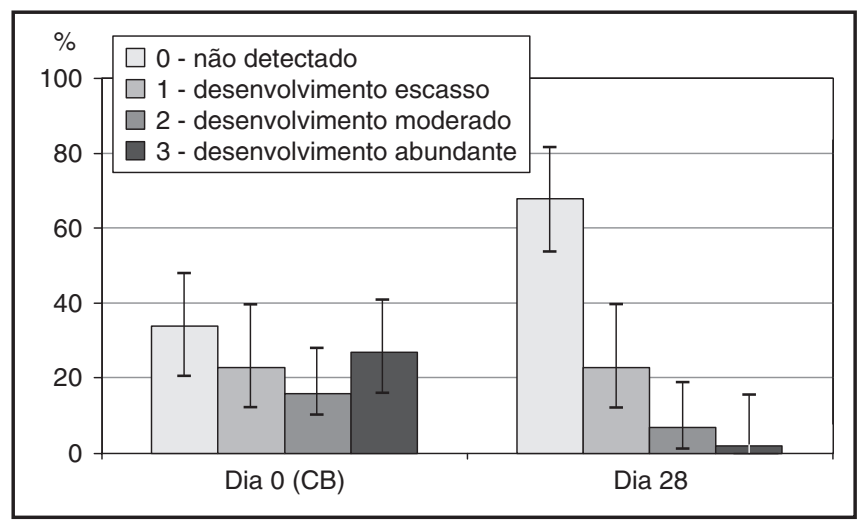

GRÁFICO 7 - Distribuição da frequência de Prevotella intermedia/nigrescens da placa subgengival identificados por cultivo. $\mathrm{CB}=$ condição basal.

diminuição do grau de desenvolvimento 3 e 2 (desenvolvimento abundante e moderado), e um aumento do grau 0 (não detectado) (Gráfico 7). Não foram registradas mudanças na composição de Pg, 
MOREIRA, A. N.; CANIGGIA, L. F.; FERREIRA, R. C.; VERÓNICA, C.; ALONSO, C.; PIOVANO, S. Efeito do controle da placa supragengival sobre a microflora subgengival e tecidos periodontais. Pesqui Odontol Bras, v. 15, n. 2, p. 119-126, abr./jun. 2001.

Aa e Fusobacterium nucleatum e peptostreptococos, no final do período experimental.

\section{DISCUSSÃO}

No presente estudo, foram examinados os indicadores clínico-microbiológicos de sítios de pacientes com diagnóstico de periodontites moderada e severa introduzidos em um programa de controle de placa supragengival.

Os indicadores clínicos foram registrados em toda a boca dos pacientes. O sítio onde foi realizado o exame microbiológico foi utilizado como unidade experimental.

A remoção de placa supragengival resultou em diminuição do índice de placa, índice gengival, sangramento a sondagem e profundidade a sondagem. A redução de PS neste trabalho foi de 0,66 \pm 0,25 .

Estes resultados concordam com os de BADERSTEN et al. ${ }^{1,2}$ (1981, 1984), que mostraram uma redução de inflamação e da profundidade a sondagem entre 0,5 e $0,8 \mathrm{~mm}$.

Neste trabalho, com relação ao efeito sobre a composição da microflora subgengival, foi observada, por cultivo, diminuição significativa de $\mathrm{Pi} / \mathrm{n}$. Por MCE, um aumento de células cocóideas e diminuição de treponemas pequenos.

Outros investigadores têm observado que a microflora subgengival pode ser alterada devido ao controle da placa supragengival quando a PS é de 4 a $5 \mathrm{~mm}^{4,14}$.

Com profundidade a sondagem $\geq 6$, KHO et al. ${ }^{6}$ (1985) e BELTRAMI et al. ${ }^{3}$ (1987) não registraram impacto sobre a microflora subgengival.

As diferenças entre os estudos poderiam estar associadas com a severidade da enfermidade, a freqüência e profundidade na remoção da placa supragengival e o método empregado para a coleta da placa subgengival.

Os treponemas pequenos, no final do período experimental, registraram ausência de sítios com proporções $\geq 15 \%$ e uma tendência a aumento nos sítios com proporções $<2 \%$.

Os resultados obtidos indicaram que a redução de IG, SS e PS poderia ser devida a uma alteração da microflora supragengival ou a variações na microflora subgengival ou ambas, que possibilitaria uma melhora ou redução da inflamação na margem gengival.

Com referência ao cultivo, não foram registradas mudanças na composição de Aa, Pg, Fusobac- terium nucleatum e peptostreptococos no final do período experimental.

Outros investigadores ${ }^{16}$ têm mostrado a dificuldade de eliminar Aa com terapia mecânica subgengival, o que leva a crer que um controle mecânico supragengival seria, todavia, ainda mais ineficaz, o que confirmaria os resultados obtidos neste trabalho.

$\mathrm{Pi} / \mathrm{n}$ registrou uma diminuição do grau de desenvolvimento 3 e 2 e um aumento do grau 0 .

As mudanças na microflora subgengival podem ser devidas à remoção da placa subgengival durante a coleta com cureta para observação por MCE e o ensino e avaliação de higiene bucal simultâneos.

MOUSQUES et $a .^{18}$ (1980) têm descrito que a coleta de amostras de placa subgengival pode alterar o ecossistema nessa região.

Nas condições experimentais deste trabalho, foi observada menor prevalência de Pg na condição basal (18\%) em relação à registrada por outros investigadores ${ }^{14}$.

Alguns parâmetros clínicos e microbiológicos poderiam ser modificados com o controle da placa supragengival, entretanto a saúde dos tecidos periodontais não seria alcançada.

É necessário maior número de estudos, com protocolos padronizados, um maior número de pacientes e por períodos experimentais maiores para determinar o efeito do controle da placa supragengival sobre o ecossistema subgengival e os tecidos periodontais.

\section{CONCLUSÕES}

De acordo com os resultados obtidos, pôde-se inferir as seguintes conclusões:

- no final do período experimental, foram registradas diferenças significativas no indice de placa, gengival, SS e PS nos sítios analisados;

- os morfotipos microbianos mostraram uma diminuição significativa de treponemas pequenos e um aumento significativo de células cocóideas;

- dos microorganismos da placa subgengival estudados por cultivo, não se observaram diferenças significativas de Aa, Pg, Fusobacterium nucleatum e peptostreptococos no final do período experimental;

- $\mathrm{Pi} / \mathrm{n}$ evidenciou diminuição significativa no final do período experimental a partir das amostras de placa subgengival. 
MOREIRA, A. N.; CANIGGIA, L. F.; FERREIRA, R. C.; VERÓNICA, C.; ALONSO, C.; PIOVANO, S. Efeito do controle da placa supragengival sobre a microflora subgengival e tecidos periodontais. Pesqui Odontol Bras, v. 15, n. 2, p. 119-126, abr./jun. 2001.

MOREIRA, A. N.; CANIGGIA, L. F.; FERREIRA, R. C.; VERÓNICA, C.; ALONSO, C.; PIOVANO, S. Effect of supragingival plaque control on subgingival microflora and periodontal tissues. Pesqui Odontol Bras, v. 15, n. 2, p. 119-126, abr./jun. 2001.

The aim of this study was to investigate, clinically and microbiologically, forty-four sites in 11 patients presenting with generalized chronic periodontitis. Plaque Index (PI), Gingival Index (GI), Probing Bleeding (PB), Probing Depth (PD) and Insertion Level (IL) were registered. Samples of subgingival plaque were collected in the same sites for cultivation of anaerobic bacteria and determination of microbiological morphotypes using dark field microscopy. Clinical and microbiological data were recorded on the baseline and 4 weeks after the adoption of a program to control supragingival plaque and calculus. The microbiological analysis categorized the degree of development as follows: 0 - not detected, 1 - scarce, 2 - moderate and 3 - abundant. The clinical results at the baseline and on the $28^{\text {th }}$ day were, respectively: PI - $1.73 \pm 0.10$ and $0.30 \pm 0.08$; GI $-1.73 \pm 0.08$ and $1.41 \pm 0.08$; PB $-0.91 \pm 0.04$ and $0.59 \pm 0.07$; PD $-6.43 \pm 0.20$ and $5.77 \pm 0.25$; and IL $-6.86 \pm 0.32$ and $6.52 \pm 0.34$. There was significant decrease in PI, GI, PB and PD. However, the difference in IL was not significant. The relative proportions of the microbial morphotypes observed under dark field microscopy at the baseline and on the $28^{\text {th }}$ day were, respectively: coccoid cells $-21.16 \pm 3.77$ and $36.00 \pm 4.66$; mobile bacillus $-44.86 \pm 2.65$ and $39.50 \pm 2.64$; and total treponemes $-24.66 \pm 3.08$ and $19.25 \pm 2.75$. The cultures presented, at the baseline and on the $28^{\text {th }}$ day, respectively: Prevotella intermedia/nigrescens $(\mathrm{Pi} / \mathrm{n})-1.36 \pm 0.18$ and $0.43 \pm 0.11$; Porphyromonas gingivalis - $0.48 \pm 0.16$ and $0.32 \pm 0.13$; Actinobacillus actinomycetemcomitans $-0.23 \pm$ 0.09 and $0.23 \pm 0.10$; Fusobacterium nucleatum $-0.32 \pm 0.14$ and $0.41 \pm 0.13$; and peptostreptococci $-0.82 \pm 0.19$ and $0.54 \pm 0.16$. There was a significant increase in the number of coccoid cells and a decrease in the number of treponemes and $\mathrm{Pi} / \mathrm{n}$.

UNITERMS: Periodontics; Microbiology.

\section{REFERÊNCIAS BIBLIOGRÁFICAS}

1. BADERSTEN, A.; NILVÉUS, R.; EGELBERG, J. Effect of nonsurgical periodontal therapy I: moderately advanced periodontitis. J Clin Periodontol, v. 8, p. 57-72, 1981.

2. BADERSTEN, A.; NILVÉUS, R.; EGELBERG, J. Effect of nonsurgical periodontal therapy II: severely advanced periodontitis. J Clin Periodontol, v. 11, p. 63-76, 1984.

3. BELTRAMI, M.; BICKEL, M.; BAEHNI, P. C. The effect of the supragingival plaque control on the composition of subgingival microflora in human periodontitis. J Clin Periodontol, v. 14, p. 161-164, 1987.

4. DAHLÉN, G.; LINDHE, J.; SATO, K. et al. The effect of supragingival plaque control on the subgingival microbiota in subjects with periodontal disease. J Clin Periodontol, v. 19, p. 802-809, 1992.

5. KATSANOULAS, T.; RENEE, L.; ATTSTRÖM, R. The effect of supragingival plaque control on the composition of the subgingival flora in periodontal pockets. J Clin Periodontol, v. 19, p. 760-765, 1992.

6. KHO, P.; SMALES, F. C.; HARDIE, J. M. The effect of supragingival plaque control on the subgingival microflora. $\mathbf{J}$ Clin Periodontol, v. 12, p. 676-686, 1985.

7. LISON, L. Estadística aplicada a la biologia experimental. Buenos Aires : Eudeba Manuales, 1976.

8. LISTGARTEN, M. A. Microbiological testing in the diagnosis of periodontal disease. J Periodontol, v. 63, p. 332-337, 1992.

9. LISTGARTEN, M. A. Subgingival microbiological differences between periodontally healthy sites and diseased sites prior to and after treatment. Int $\mathbf{J}$ Periodontics Restorative Dent, v. 4, p. 27-33, 1984.
10. LISTGARTEN, M. A.; HELLDEN, L. Relative distribution of bacteria at clinically healthy and periodontally diseased sites in humans. J Clin Periodontol, v. 5, p. 115-119, 1978.

11. LISTGARTEN, M. A.; SCHIFTER, C. Differential dark field microscopy of subgingival bacteria as an aid in selecting recall intervals: results after 18 months. J Clin Periodontol, v. 9, p. 305-316, 1982.

12. LÖE, $H$. The gingival index, the plaque index and retention index system. J Periodontol, v. 38, p. 610-616, 1967.

13. LOSS, B.; CLAFFEY, N.; CRIGGER, M. Effect of oral hygiene measures on clinical and microbiological parameters of periodontal disease. J Clin Periodontol, v. 15, p. 211-216, 1988.

14. MAC NABB, H.; MOMBELLI, A.; LANG, N. P. Supragingival cleaning 3 times a week. The microbiological effects in moderately deep pockets. J Clin Periodontol, v. 19, p. 348-356, 1992.

15. MÖLLER, A. J. Microbiological examination of root canals and periapical tissues of human teeth. Methodological studies. Odontol Tidskr, v. 20, n. 5, 1966.

16. MOMBELLI, A.; GMÜR, R.; GOBBI, C.; LANG, N. P. Actinobacillus actinomycetemcomitans in adult periodontitis II: topographic distribution before and after treatment. J Periodontol, v. 65, p. 820-826, 1994.

17. MOUSQUES, T.; LISTGARTEN, M. A.; PHILliPS, R. W. Effect of scaling and root planning on the composition of the human subgingival microbial flora. J Periodontal Res, v. 15, p. 144-151, 1980.

18. MOUSQUES, T.; LISTGARTEN, M. A.; STOLLER, N. H. Effect of sampling on the composition of the human 
MOREIRA, A. N.; CANIGGIA, L. F.; FERREIRA, R. C.; VERÓNICA, C.; ALONSO, C.; PIOVANO, S. Efeito do controle da placa supragengival sobre a microflora subgengival e tecidos periodontais. Pesqui Odontol Bras, v. 15, n. 2, p. 119-126, abr./jun. 2001.

subgingival microbial flora. J Periodont Res, v. 15, p. 137-143, 1980.

19. NOGUEIRA MOREIRA, A. Evaluación de antimicrobianos en el tratamiento de la enfermedad periodontal. Buenos Aires, 1999. 168 p. Tese (Doutorado) Faculdad de Odontologia, Universidad de Buenos Aires.

20. RAMFJORD, S. P. Indices for prevalence of periodontal disease. J Periodontol, v. 30, p. 51-59, 1959.

21. RAMFJORD, S. P.; CAFFESE, R.; MORRISON, E. C. et al. Four modalities of periodontal treatment compared over 5 years. J Clin Periodontol, v. 14, p. 445-452, 1987.

22. RAMS, T. E.; LISTGARTEN, M. A.; SLOTS, J. Utility of 5 major putative periodontal pathogens and selected clinical parameters to predict periodontal breakdown in patients on maintenance care. J Clin Periodontol, v. 23, p. 346-354, 1996.

23. SLOTS, J. Selective medium for isolation of Actinobacillus actinomycetemcomitans. J Clin Periodontol, v. 15, p. 606-609, 1982.

24. SLOTS, J.; FEIK, D.; RAMS, T. E. Actinobacillus actinomycetemcomitans and Bacteroides intermedius in human periodontitis: age relatioship and mutual association. J Clin Periodontol, v. 17, p. 659-662, 1990.

25. SMULOW, J. B.; TURESKY, S. S.; HILL, R. G. The effect of supragingival plaque removal on anaerobic bacteria in deep periodontal pockets. J Am Dent Assoc, v. 107, p. 737-742, 1983.

26. SUMMANEN, P.; BARON, E. J.; CITRON, D. M. et al. Wdasworth anaerobic bacteriology manual, 5. ed. Belmont : California Star Publishing, 1993.

27. WOLFF, L.; DAHLEN, G.; AEPPLI, D. Bacteria as risk markers for periodontitis. J Periodontol, v. 64, p. 498-510, 1994.

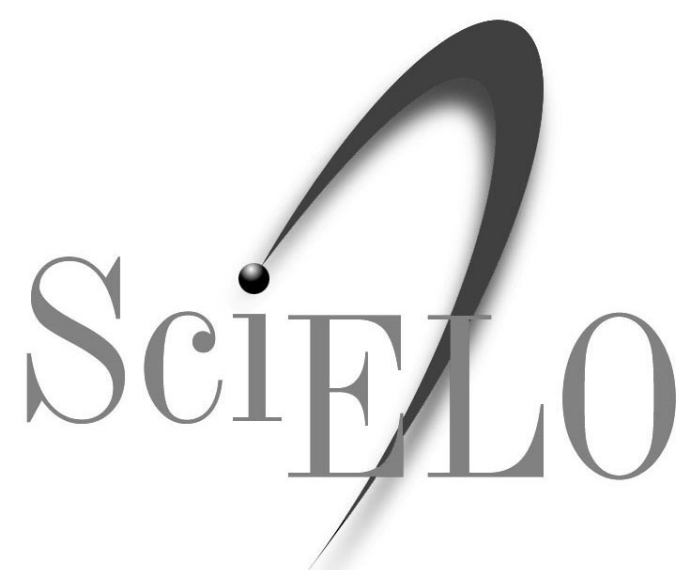

\section{Scientific Electronic Library Online http://www.scielo.br Parceria: FAPESP/BIREME}

SciELO - Scientific Electronic Library Online é uma coleção eletrônica de revistas científicas disponíveis na Internet. A coleção traz os textos completos de artigos científicos, bases de dados e relatórios de uso e de impacto da literatura científica publicada no site.

Em operação desde 1997, atualmente estão disponíveis 29 revistas brasileiras nas áreas de ciências biológicas e da saúde, ciências sociais e humanas, ciências agrárias, física etc. Novas publicações são incluídas regularmente em várias especialidades.

Entre as revistas de nossa coleção, está a Pesquisa Odontológica Brasileira.

$$
\begin{aligned}
& \text { Consulte a Pesquisa Odontológica Brasileira (Revista de Odontologia da } \\
& \text { USP) na SciELO - http://www.scielo.br/rousp }
\end{aligned}
$$

